\title{
Universal electronic-cigarette test: physiochemical characterization of reference e-liquid
}

\author{
Jeffrey J. Kim ${ }^{1 *}$, Nicole Sabatelli ${ }^{2}$, Wojtek Tutak ${ }^{1,6}$, Anthony Giuseppetti ${ }^{1}$, Stanislav Frukhtbeyn ${ }^{1}$, Ian Shaffer ${ }^{3}$,
} Joshua Wilhide ${ }^{3}$, Denis Routkevitch ${ }^{4}$ and John M. Ondov ${ }^{5}$

\begin{abstract}
Background: Despite the rising health and safety concerns of e-cigarettes, a universal e-cigarette testing method is still in its early developmental stage. The aim of this study was to develop an e-liquid Reference Material that can be used to improve accuracy and reproducibility of research results, and advance health risk assessment of e-cigarette products.

Methods: E-liquid Reference Material was developed by purity assessment, gravimetric measurement, homogeneity testing, and stability testing with material and instrument traceability (adopted from ISO 35:2006E).

Results: Homogeneity tests showed e-liquid Reference Material requires $\geq 1 \mathrm{~h}$ rotation at a speed of $5 \mathrm{rpm}$ to reach complete homogeneity. Stability tests showed homogeneity is intact for at least 2 weeks without secondary separation, and e-liquids are stable in $21^{\circ} \mathrm{C}-50^{\circ} \mathrm{C}$ thermocycling conditions up to $72 \mathrm{~h}$. A change in the e-liquid color was first observed at day seven, and progressed to 2 - and 16 - fold increase in absorbance by one and 6 months respectively. We found that e-liquids do not have inherent material instabilities such as immiscibility or secondary separation. However, discrepancies in concentration and composition arose mainly due to viscosity of propylene glycol and glycerin. Aerosol generated from the e-liquid Reference Material had 16 chemical-byproducts and was composed of $\sim 634,000$ particles of which 38\% were Fine Particulate Matters ( $<0.5 \mu \mathrm{m}$ in diameter).

Conclusions: The efforts described here to create a standardized e-liquid Reference Material aim to provide unbiased and robust testing parameters that may be useful for researchers, the industry and government agencies. Additionally, the reference e-liquid could open a channel of conversation among different laboratories by providing the means of independent verification and validation while establishing a system of transparency and reproducibility in materials and methods.
\end{abstract}

Keywords: Electronic-cigarette, e-cig, Reference material, e-liquid, Universal testing method

\section{Background}

The electronic cigarette industry has been doubling its annual growth, overtaking combustible cigarette sales among U.S. young adults in 2014; its global projected sales will reach $\$ 10$ billion by $2017[1,2]$. The latest studies show $79 \%$ of U.S. consumers recognize e-cigarettes and $44 \%$ believe e-cigarettes are less harmful than traditional cigarettes despite warnings from the World Health

\footnotetext{
*Correspondence: jeffrey.kim@nist.gov

${ }^{1}$ Clinical Research, Volpe Research Center, ADA Foundation, 100 Bureau Drive Stop 8546 NIST, Gaithersburg, MD 20899, USA

Full list of author information is available at the end of the article
}

Organization (WHO), U.S. Food and Drug Administration (FDA), Centers for Disease Control and Prevention (CDC), American Medical Association, American Lung Association, and American Dental Association [3-9]. In 2014, $12.6 \%$ of U.S. and $11.6 \%$ of European adults have tried e-cigarettes at least once $[10,11]$. France had the highest number of adult e-cigarette users (21.3\%), while Portugal had the lowest (5.7\%) [11]. There are approximately 9 and 2.1 million regular e-cigarette users in the U.S. and the U.K., respectively $[10,12]$. The success of the e-cigarette industry, in part, can be attributed to aggressive marketing that targets specific age groups and the public's 
perception that e-cigarettes are a safer alternative to traditional tobacco products $[13,14]$.

In the U.S., over 400 companies distribute thousands of products through local "vape shops" and online stores with little or no regulation [15]. Most products are imported from China which is the largest manufacturer of ecigarettes, producing $95 \%$ of the world's e-cigarettes, primarily for the European and the U.S. consumer markets [16]. Most companies do not disclose ingredients in eliquids or provide proof of safety, good manufacturing practices and/or quality control measures [17-19]. Several studies have reported significant differences between nicotine concentrations indicated by manufacturers vs. the actual concentrations verified by independent laboratories [2026]. More concerning is the detection of various levels of nicotine in "nicotine-free" e-liquids [21, 22, 26]. In addition, unapproved pharmaceutical ingredients - metals, carcinogens, toxic chemicals, and industrial grade propylene glycol - have been identified in commercially available e-liquids $[27,28]$. In 2014, the Australia Department of Health implemented strict regulations under the Liquid Nicotine and Personal Importation for Use in E-cigarettes Guideline [29]. In 2016, European Union Member States started to regulate e-cigarettes as part of the EU Tobacco Products Directive [30]. In 2016, the U.S. Food and Drug Administration amended the 2009 Family Smoking Prevention and Tobacco Control Act by extending its authority over e-cigarettes, cigars and all other tobacco products [31]. The effectiveness of policy implementation on public health and its impact on the e-cigarette industry remain to be seen.

Many contributing factors to inconsistent and contradicting findings in e-cigarette research include lack of standardized research materials, testing devices and test methods. These inadequacies remain a major hurdle in bringing clarity to the situation $[19,25,32]$. Considering there are over a thousand e-liquid formulations, many with significant quality variations, it is not feasible to test every product. This challenge is not new to the manufacturing industry. Manufacturers have applied various quality control practices to improve the manufacturing process and product quality. One of the most common methods is implementing a Reference Material (RM) in the quality control process. RM is a matrix-matched material with assigned target values and assigned ranges for each variable, reliably determined from data obtained by repeated analysis [33]. RMs are routinely used to check the quality and metrological traceability of products, as well as for instrument calibration. The Reference Cigarettes produced by the Center for Tobacco Reference Products (University of Kentucky), for example, have provided much needed standards for tobacco manufacturers, government agencies and research institutions. Similar reference products have not been available in the e-cigarette research field until now. Recently, the British Standards Institution (BSI) and Association Française de Normalisation (AFNOR), with support from tobacco product manufacturers and the electronic cigarette industry trade association, published PAS 54115:2015 and XP D 90-300-2 respectively. Both technical specifications explain aspects of manufacturing standards and analytical testing methods in detail mainly from the industry perspective. However, development and utility of e-liquid RM was not explicitly discussed in these specifications.

In this study, we describe development of an e-liquid RM and physiochemical characterization of aerosol in support of establishing universal e-cigarette testing parameters. The e-liquid RM can serve as a key component in the proper experimental design process to improve accuracy, transparency, and reproducibility of data. For example, current e-cigarette studies use combustible cigarettes, Nicorette gums, nicotine inhalers and/or lozenges as a control [1]. Although their use is well justified for comparative analyses (e.g. the amount of harmful and potentially harmful constituents (HPHCs) in e-cigarettes vs. combustible cigarettes), e-liquid RM is necessary for two reasons: (1) the chemical composition and delivery process are unique in e-cigarettes compared to other nicotine and tobacco products which makes direct comparison between the two difficult, and (2) the quality of e-liquids and e-cigarette devices vary so widely that researching with less-reliable commercial products will inevitably lead to inconsistent final outcomes. The e-liquid RM will allow users to check the quality of unknown e-liquids, perform instrument calibration, assess toxicological risks, and test safety and efficacy of e-cigarette devices.

Currently, there is considerable variance in e-liquids and in testing devices. With so many variables it is difficult to perform meaningful studies that permit comparison of results among independent laboratories. This study aims to develop an e-liquid RM that can be used to compare different e-cigarette devices and e-liquids, assess various testing parameters, and improve reproducibility in e-cigarette research.

\section{Methods \\ E-liquid Reference Material (RM) \\ Starting materials with specified properties}

E-liquid RM was prepared by combining propylene glycol, glycerin and nicotine. Propylene glycol (Sigma Aldrich 49770) and glycerin (Sigma Aldrich 82280) were puriss. p.a., analytical grade chemicals that met American Chemical Society (ACS) specifications. They were tested to be $>99.9 \%$ pure by Gas Chromatography (GC) and can be traced by Lot Numbers SZBE279CV and BCBN5225V, respectively. Nicotine solution (Sigma Aldrich N3876) was tested to be 99.5\% pure (Lot Number 1449194 V) (Fig. 1). 


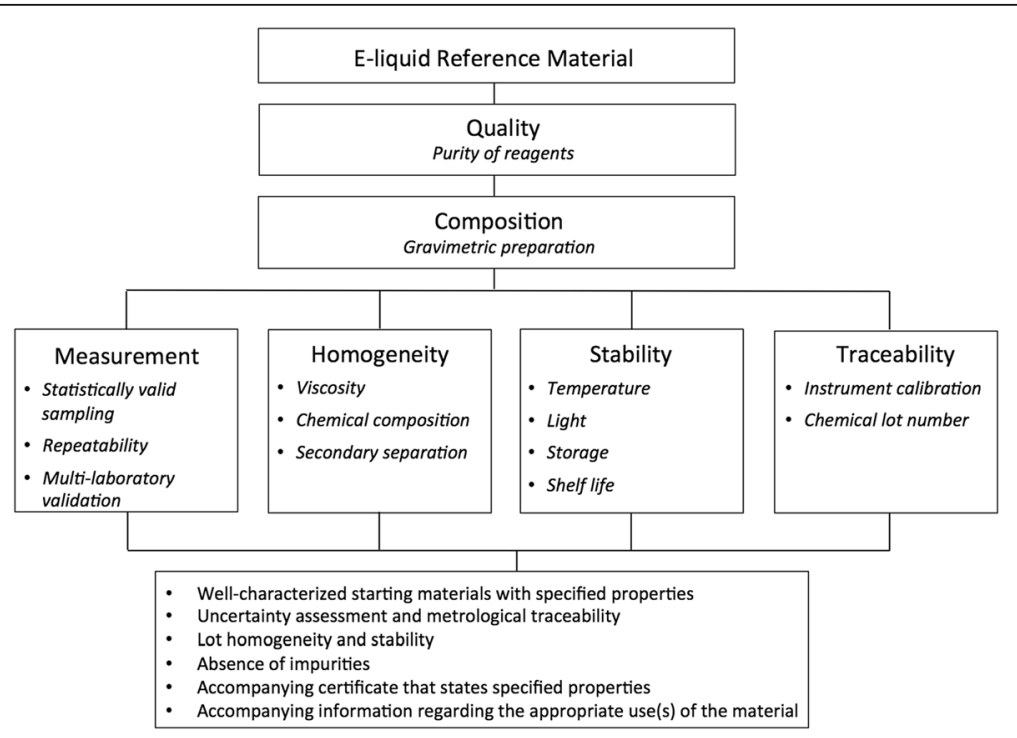

Fig. 1 A Standard Operating Procedure (SOP) for e-liquid Reference Material formulation by adopting ISO Guide 35:2006E (Reference Materials: General and Statistical Principles for Certification) guideline. The reference e-liquid was characterized by measuring quality, homogeneity, stability and traceability of each component of the e-liquid

\section{Gravimetric preparation}

An analytical micro-scale (A\&D) was used to weigh $1.036 \mathrm{~g}$ propylene glycol, $1.250 \mathrm{~g}$ glycerin and $20.0 \mathrm{mg}$ nicotine to make the reference e-liquid (1:1 propylene glycol/glycerin (v/v) with $10 \mathrm{mg} / \mathrm{ml}$ nicotine) as shown in Fig. 1. 8:2 and 2:8 propylene glycol/glycerin e-liquid RMs were prepared by weighting $1.650 \mathrm{~g}$ propylene glycol, $0.500 \mathrm{~g}$ glycerin and $20.0 \mathrm{mg}$ nicotine; and weighting $0.410 \mathrm{~g}$ propylene glycol, $2.000 \mathrm{~g}$ glycerin and $20.0 \mathrm{mg}$ nicotine, respectively.

\section{Homogeneity tests}

Within-bottle homogeneity (based on the ISO 35:2006E guideline) was measured by rotating $1 \mathrm{~mL}$ e-liquid $\mathrm{RM}$ in a $7.5 \mathrm{~mL}$ round-bottom test tube $(13 \times 100 \mathrm{~mm}$, Pyrex $)$ using a laboratory rotator (230401 V, VWR) set at 5 revolutions per min (r.p.m.) (Fig. 1). At 5 min, 20 min, 1 h, 3 h and $6 \mathrm{~h}$ timepoints, three subsamples were collected from the e-liquid RM and saved for nicotine concentration measurement (Table 1). Next, secondary separation was measured by leaving a homogenous 1:1 propylene glycol/ glycerin e-liquid RM undisturbed for $24 \mathrm{~h}$ and 2 weeks (Table 1).

\section{Stability tests}

For stability tests, three parameters of the e-liquid RM were considered: range of temperature, change in nicotine concentration, and color stability (Fig. 1). The temperature of the e-liquid RM was measured at rest and immediately following $5 \mathrm{~min}$ of e-cigarette use in our operating conditions ( $2.8 \Omega$ heating element at $3.6 \mathrm{~V}$ : total of $4.63 \mathrm{~W}$ ) using a digital thermometer. To assess stability of the e-liquid RM under the temperature change, 1:1 propylene glycol/glycerin e-liquid RM was exposed to $21 \mathrm{C}^{\circ}$ (at rest temperature) for $90 \mathrm{~s}$ and $50 \mathrm{C}^{\circ}$ (after $5 \mathrm{~min}$ of use) for $90 \mathrm{~s}$ over 24 and $72 \mathrm{~h}$ time periods by using a thermocycler. Three subsamples were collected from the e-liquid RM and saved for nicotine concentration measurement (Table 1). The color change of the e-liquid RM was quantified by measuring absorbance (490 $\mathrm{nm})$ using a spectrophotometer at day 0,1 month, and 6 months from the initial preparation date (Table 1).

\section{Absence of metal impurities in the reference e-liquid}

To show purity, the e-liquid RM was analyzed for metal contaminants using Prodigy-Spec ICP-OES (Teledyne Leeman Labs) according to the manufacturer's protocol. Testing parameters were as follows: Temperature $=34{ }^{\circ} \mathrm{C}$, Torch gas coolant = 19.9 LPM, Aux =0.31 LPM, Neubulizer $=34.2 \mathrm{PSI}$, and Pump $=1.4 \mathrm{mM} / \mathrm{min}$. The concentrations of eight metals (cadmium, chromium, cobalt, copper, lead, manganese, nickel, and palladium) were measured in all e-liquid RM samples.

\section{Nicotine concentration}

Nicotine concentration was measured by the previously established protocol using a HPLC-UV (Agilent, Zorbax column) [34]. Measurement parameters were as follows: Injection volume $=100 \mathrm{uL}$, Temperature $=25^{\circ} \mathrm{C}$, Flow rate $=1 \mathrm{~mL} / \mathrm{min}$, Wavelength $=262 \mathrm{~nm}, \quad$ Retention $=4.3-$ $4.5 \mathrm{~min}$. A standard calibration curve was calculated using $0,3,10$ and $30 \mu \mathrm{g} / \mathrm{mL}$ nicotine. 
Table 1 E-liquid RM homogeneity and stability tests ${ }^{\mathrm{a}}$

\begin{tabular}{|c|c|c|c|c|c|}
\hline Variable & Sub-sample 1 & Sub-sample 2 & Sub-sample 3 & S.D. & \# of liquid phase \\
\hline \multicolumn{6}{|c|}{ Nicotine concentration $(\mathrm{mg} / \mathrm{ml})$} \\
\hline \multicolumn{6}{|c|}{ Homogeneity test (1:1) } \\
\hline $5 \mathrm{~min}$ & 10.65 & 9.19 & 8.93 & 0.92 & 2 \\
\hline $20 \mathrm{~min}$ & 9.90 & 9.79 & 9.55 & 0.17 & 2 \\
\hline $1 \mathrm{~h}$ & 9.69 & 10.00 & 9.78 & 0.15 & 1 \\
\hline \multicolumn{6}{|c|}{ Homogeneity test (8:2) } \\
\hline $5 \mathrm{~min}$ & 10.41 & 10.29 & 10.23 & 0.31 & 2 \\
\hline $20 \mathrm{~min}$ & 10.48 & 10.53 & 11.82 & 0.66 & 2 \\
\hline $1 \mathrm{~h}$ & 10.89 & 10.61 & 11.00 & 0.20 & 1 \\
\hline \multicolumn{6}{|c|}{ Homogeneity test (2:8) } \\
\hline $5 \mathrm{~min}$ & 9.28 & 8.49 & 7.98 & 0.59 & 2 \\
\hline $20 \mathrm{~min}$ & 9.63 & 8.44 & 9.63 & 0.64 & 2 \\
\hline $1 \mathrm{~h}$ & 9.73 & 9.36 & 8.33 & 0.73 & 1 \\
\hline $3 \mathrm{~h}$ & 9.54 & 8.91 & 9.14 & 0.32 & 1 \\
\hline $6 \mathrm{~h}$ & 10.99 & 9.88 & 11.18 & 0.70 & 1 \\
\hline \multicolumn{6}{|c|}{ Stability test (1:1) (thermocycler) } \\
\hline $24 \mathrm{~h}$ & 9.97 & 10.02 & 10.00 & 0.02 & 1 \\
\hline $72 \mathrm{~h}$ & 9.98 & 10.04 & 10.05 & 0.03 & 1 \\
\hline \multicolumn{6}{|c|}{ Absorbance $(490 \mathrm{~nm})$} \\
\hline \multicolumn{6}{|c|}{ Stability test (1:1) (spectrophotometer) } \\
\hline Day 0 & 11.99 & 12.12 & 12.13 & 1 & 1 \\
\hline 1 month & 26.29 & 26.08 & 25.95 & 2.14 & 1 \\
\hline 6 month & 200.84 & 198.28 & 188.69 & 16.20 & 1 \\
\hline
\end{tabular}

${ }^{a} 1: 1$ propylene glycol/glycerin $=50 \%$ propylene glycol and $50 \%$ glycerin by volume prepared using mass

$8: 2$ propylene glycol/glycerin $=80 \%$ propylene glycol and $20 \%$ glycerin by volume prepared using mass

$2: 8$ propylene glycol/glycerin $=20 \%$ propylene glycol and $80 \%$ glycerin by volume prepared using mass

\section{E-cigarette testing device}

The main testing unit was designed and built by researchers at the ADA Foundation Volpe Research Center (Gaithersburg, MD), in collaboration with researchers at the University of Maryland (College Park, MD) using a custom acrylonitrile butadiene styrene (ABS) enclosure $(6 " \times 4 " \times 2 ")$ with a 510 adaptor, a precision wattage meter and power analyzer (Guanglian Town 'G.T.' power), and an independent $9 \mathrm{~V}$ DC power source. Each component of the main unit was carefully selected from lead-free materials including wires and solder. The main unit has two settings: (1) the e-cigarette user mode and (2) the research mode. In the e-cigarette user mode, power is delivered by a $3.6 \mathrm{~V}$ lithium-ion battery similar to how e-cigarette users would use the device as described in e-cigarette online forums. In the research mode, filtered power is delivered by a DC power supply (Model D-612 T, Electro Products). The operator is able to increase or decrease the voltage delivered to the main unit in $0.2 \mathrm{~V}$ increments. Real-time voltage $(\mathrm{V})$, current $(\mathrm{A})$, and power $(\mathrm{W})$ are displayed on a LED screen on the main unit. We used a $2.0 \mathrm{ml}$ Vivi Nova tank system with a heating element for the proof-of- principle experiments described here. Based on the resistance of the heating element, we used a conservative power setting of $3.6 \mathrm{~V}$ (total of $4.63 \mathrm{~W}$ based on $P=\mathrm{V}^{2} \mathrm{x}$ R) determined by online "vaping power charts" for all of our experiments. Prior to running the e-cigarette, we made sure that the wick was fully saturated with e-liquid by allowing $\geq 15$ min incubation period after each fill.

\section{E-cigarette aerosol generation}

We used two different methods to capture e-cigarette aerosol depending on specific downstream applications: (1) chemical analysis by Gas Chromatography Mass Spectrometry (GC-MS), and (2) detection of metal(s) by Inductively Coupled Plasma - Optical Emission Spectrometry (ICP-OES).

To capture e-cigarette aerosol for GC-MS analysis, we extracted aerosol directly by connecting a $50 \mathrm{~mL}$ glass syringe (Micro-mate) to the e-cigarette mouthpiece. $100 \mathrm{~mL}$ e-cigarette aerosol was immediately injected into a $22 \mathrm{~mL}$ GC headspace vial with Polytetrafluoroethylene (PTFE)/ Silicone rubber septa (Perkin Elmer) using an 18 gauge needle. We used $1 \mathrm{~cm}^{3}$ GC glass wool (Sigma Aldrich 20384) 
as a filter between the mouthpiece and the syringe tip to prevent inadvertent e-liquid aspiration into the syringe space which can lead to clogged GC inlets.

To determine presence of metal(s), we collected the ecigarette aerosol (15 puffs or 150 puffs) in $30 \mathrm{~mL}$ of deionized water (minimum volume allowed) using a gas condenser (Pyrex 1760-125). To prepare for ICP-OES analyses, we prepared $2 \% \mathrm{HNO}_{3}$ samples by adding $0.35 \mathrm{~g}$ of $69 \% \mathrm{HNO}_{3}$ to $11.65 \mathrm{~g}$ of the deionized water containing e-cigarette aerosol. The presence of eight metals that are commonly found in heating elements was evaluated: cadmium $(\mathrm{Cd})$, chromium $(\mathrm{Cr})$, cobalt $(\mathrm{Co})$, copper $(\mathrm{Cu})$, lead $(\mathrm{Pb})$, manganese $(\mathrm{Mn})$, nickel $(\mathrm{Ni})$, and palladium $(\mathrm{Pd})$. The final results are shown in concentration $(\mathrm{mg} / \mathrm{L})$ after considering the dilution factor of the collecting liquid medium.

In this study, all tests were performed with the published physiological human e-cigarette puffing topography: $50 \mathrm{~mL}$ puff volume in $4 \mathrm{~s}$ puff duration every $18 \mathrm{~s}$ [35]. Unless otherwise mentioned, all experiments were performed at a constant laboratory temperature $\left(21^{\circ} \mathrm{C}\right)$.

\section{Chemical characterization of e-cigarette aerosol}

All chemical measurements were performed using PerkinElmer Clarus 680 GC with MS Detection (PerkinElmer, Waltham, MA) fitted with a Velocity DB 5 column (PerkinElmer N9306325). Testing parameters of the GC method were as follows: Sampling method $=$ manual headspace, Inlet temperature $=210{ }^{\circ} \mathrm{C}$, Carrier gas $=1.43 \mathrm{~L} / \mathrm{min}$, Split $=1: 5$, Temperature ramp $=$ initial: $40{ }^{\circ} \mathrm{C}$, hold $3 \mathrm{~min}$, $6{ }^{\circ} \mathrm{C} / \mathrm{min}$ to $300{ }^{\circ} \mathrm{C}$, hold for $3 \mathrm{~min}$, and Total analysis time $=49.33 \mathrm{~min}$. Testing parameters for the MS method were as follows: MS detector $=$ PerkinElmer Clarus, ionization source $=\mathrm{El}$, Polarity $=$ positive, Mass range $=(44$ to 600$) \mathrm{m} /$ $\mathrm{z}$, Acquisition type $=$ centroid, Solvent delay $=(0.00$ to 2.00$)$ min, and Analysis time $=(2.00$ to 49.30$) \mathrm{min}$.

\section{Physical characterization of e-cigarette aerosol}

All physical measurements were performed using a TSI Aerodynamic Particle Sizer (APS 3321) according to the manufacturer's protocol. Aerosol generated by the ecigarette testing device was released into a $4 \mathrm{~L}$ glass smoking chamber by negative pressure generated by a vacuum source at $1.0 \mathrm{~L} / \mathrm{min}$. The APS sampling probe was connected directly to the smoking chamber, and was programmed to run three consecutive samples for $20 \mathrm{~s}$ with a 1 min break between each cycle.

\section{Statistical analysis}

Concentration measurement, absorbance, and metal detection were quantified using mean \pm standard deviation (S.D.) from three independent measurements. Each experiment was repeated in triplicate by calibrated operators. All statistical analyses were conducted using the MaxStat 3.6 statistical software (Jever-OT Cleverns, Germany). The significant level was established as $p<0.05$.

\section{Results \\ E-liquid Reference Material \\ Gravimetric preparation}

Gravimetric preparation of e-liquids provides for exceedingly precise compositional concentrations and ratios [36]. By implementing the Standard Operating Procedure described in Methods and Fig. 1, we demonstrated that highly accurate e-liquid RM can be formulated (a standard deviation of $0.15 \mathrm{mg}$ or less as shown in Table 1 ).

\section{Homogeneity tests}

To test uniformity of e-liquids, we examined homogeneity of the reference e-liquid by (i) measuring the time required to reach complete homogeneity and (ii) characterizing secondary separation properties and miscibility of e-liquids. We defined that the e-liquid has reached complete homogeneity when nicotine concentrations of the subsamples were statistically equal. For $1: 1$ and 8:2 propylene glycol/glycerin e-liquid RMs, we found that by $5 \mathrm{~min}$, the nicotine concentrations were not significantly different ( $t$-test, $p>0.05$ ). However, propylene glycol and glycerin still existed as two-phase at $20 \mathrm{~min}$. After $1 \mathrm{~h}$ rotation, the e-liquid was in complete homogeneity which was confirmed by the nicotine concentration and having a single liquid phase (Table 1). However, homogeneity could not be achieved for 2:8 propylene glycol/ glycerin e-liquid RM even after $6 \mathrm{~h}$ mixing based on the standard deviation fluctuation (S.D. $=0.70$ after $6 \mathrm{~h}$ ). Secondary separation measurements for $24 \mathrm{~h}$ and 2 weeks showed that composition uniformity of the eliquid stayed intact up to 2 weeks (Table 1 ).

\section{Stability tests}

E-liquids are subjected to a wide range of temperature by e-cigarette devices. We measured the temperature of the e-liquid RM sample at rest and after $5 \mathrm{~min}$ of use and found that the e-liquid in the tank was subjected to temperatures between $\left(21\right.$ to $50 \pm 3$ ) $\mathrm{C}^{\circ}$ in our operating conditions $(2.8 \Omega$ heating element at $3.6 \mathrm{~V}$ : total of $4.63 \mathrm{~W})$. Under the thermocycling conditions $\left(21 \mathrm{C}^{\circ}\right.$ for $90 \mathrm{~s}$ and $50 \mathrm{C}^{\circ}$ for $90 \mathrm{~s}$ over $24 \mathrm{~h}$ and $72 \mathrm{~h}$ time periods), we found that the subsamples remained homogenous up to $72 \mathrm{~h}$ without secondary separation compared to a control.

At day 7, the color of the e-liquid began to change from clear to yellow. The change progressed linearly with time. The absorbance $(490 \mathrm{~nm})$ of the e-liquid RM increased by 2 - and 16- fold by 1 month, and 6 months respectively compared to a control (Table 1). 


\section{Detection of metals in the reference e-liquid}

High quality RM should not contain other contaminants. To verify that the e-liquid RM is free of metal contaminants, the levels of eight metals were quantified. We found that the levels of eight metals were below the limit of detection (1 ppb by U.S. Environmental Protection Agency Method 200.8) in all eliquid RM samples.

\section{Applications}

For best results, we provide the following information regarding the appropriate use of our e-liquid Reference Material:

- Prepare the reference e-liquid immediately prior to use and store up to 1 week in room temperature, away from light and moisture.

- Use a glass round bottom vessel to homogenize. A vessel with acute ends (e.g. centrifuge tubes) traps glycerin and requires longer time to mix.

- Use minimum 6:1 vessel to liquid ratio to provide enough surface area for liquids to be mixed.

- Set a vertical rotator at $\leq 5$ r.p.m. Faster speed leads to inadequate mixing.

- RM can be customized to meet individual needs of users as long as the guiding principles described in Fig. 1 are followed. For example, some users may desire other additives such as diluents and/or flavorings in their RM. In such a case, homogeneity and stability parameters should be reestablished.

- More detailed information on development and practical usage of RMs and theories behind homogeneity and stability tests can be found in ISO Guide 35:2006(E) under chapter 6: "Evaluation measurement uncertainty", chapter 7: "Homogeneity study", chapter 8: "Stability study"; British Standards Institution (BSI) PAS 54115:2015 under chapter 4: "E-liquids"; and Association Française de Normalisation (AFNOR) XP D 90-300-2 under chapter 4: "General requirements and test methods related to e-liquids".

- Troubleshooting: typical sources of uncertainty associated with RMs are instrument effects (e.g. calibration of scale and pipettes), reagent purity, measurement conditions (e.g. temperature and humidity), operator effects (e.g. training) and random effects. When there is an observed measurement error that cannot be easily explained, one should refer to the ISO Guide to the Expression of Uncertainty in Measurement (GUM) for more information.

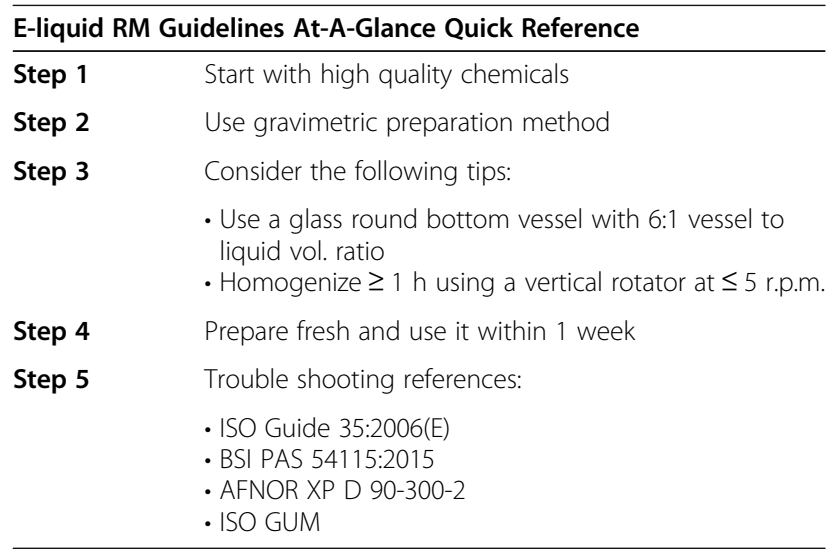

\section{Characterization of aerosol generated from the reference} e-liquid

Chemical by-products in the aerosol

When $50 \mathrm{~mL}$ aerosol generated from the reference eliquid was analyzed using GC-MS, 16 chemical byproducts (Table 2) were identified in the e-cigarette aerosol. According to the Globally Harmonized System of Classification and Labeling of Chemicals (GHS), seven chemicals were classified as "dangerous chemicals," and five carried "warning" labels. Material Safety Data Sheets (MSDS) of the chemicals showed eight may cause acute toxic effects when ingested, and eight may cause respiratory irritation at high concentration (Table 2). Chemical by-products were ranked by Signal-to-Noise $(\mathrm{S} / \mathrm{N})$ ratios based on Total Ion Chromatogram (TIC) intensity peak values.

\section{Physical properties of the reference aerosol}

Aerodynamic particle size and distribution analysis showed that the raw particle count of the reference aerosol was $634,340 \pm 164,173$ particles/sample (in $0.333 \mathrm{~L}$ ). The number of particles below $0.5 \mu \mathrm{m}$ in diameter (within Fine Particulate Matter range defined by the 2012 National Ambient Air Quality Standards for Particle Pollution, U.S. Environmental Protection Agency) was $(244,020 \pm 61,009)$ which accounts for $38 \%$ of total aerosol. The geometric mean and total concentration were $(1.40 \pm 0.06 \mu \mathrm{m})$ and $\left(1903 \pm 492\right.$ particle $\left./ \mathrm{cm}^{3}\right)$, respectively. The overall distribution is shown in Fig. 2.

\section{Presence of metals in the aerosol}

The reference aerosol was analyzed for the presence of metals in $750 \mathrm{~mL}$ (15 puffs) and $7.5 \mathrm{~L}$ (150 puffs) aerosol using Prodigy-Spec ICP-OES. The levels of the aforementioned eight metals in $750 \mathrm{~mL}$ (15 puffs) and $7.5 \mathrm{~L}$ (150 puffs) aerosol samples were below the limit of ICPOES detection when a new e-cigarette tank was used. Approximately after 4 months of testing ( $20 \mathrm{~h}$ total usage), the e-cigarette had accumulated a noticeable amount of 
Table 2 Chemical by-products found in reference aerosol

\begin{tabular}{|c|c|c|c|c|}
\hline Name & $\begin{array}{l}\text { GHS signal } \\
\text { word }\end{array}$ & Hazard classification & $\begin{array}{l}\text { CAS } \\
\text { number }\end{array}$ & $\begin{array}{l}\mathrm{S} / \mathrm{N} \text { ratio based on } \mathrm{TIC} \text { peak } \\
\text { intensity }\end{array}$ \\
\hline Nicotine & Danger & $\begin{array}{l}\text { Acute Toxicity } 2 \\
\text { Acute Aquatic Toxicity } 1 \\
\text { Chronic Aquatic Toxicity } 1 \\
\text { Fatal if swallowed or in contact with } \\
\text { skin }\end{array}$ & $54-11-5$ & 115578.89 \\
\hline Nicotyrine & Danger & $\begin{array}{l}\text { Skin Irritation } 3 \\
\text { Acute Toxicity } 3 \\
\text { Respiratory Irritation } 3\end{array}$ & $487-19-4$ & 1405.67 \\
\hline Propylene glycol & None & None & $57-55-6$ & 709.6 \\
\hline 4-Pyridinecarboxaldehyde & Warning & $\begin{array}{l}\text { Skin Irritation } 2 \\
\text { Eye Irritation 2A } \\
\text { STOT SE } 3 \\
\text { Combustible liquid } \\
\text { Harmful if swallowed } \\
\text { May cause respiratory irritation }\end{array}$ & $872-85-5$ & 74.1 \\
\hline 2,4,7-trimethyl-1,8-naphthyridine & NF & $\mathrm{NF}$ & $14757-44-9$ & 59.46 \\
\hline 1-Butanol & Danger & $\begin{array}{l}\text { Flammable liquid } 3 \\
\text { Acute Toxicity } 4 \\
\text { Skin Irritation } 2 \\
\text { Eye Damage } 1 \\
\text { STOT SE } 3 \\
\text { Harmful if swallowed } \\
\text { May cause respiratory irritation } \\
\text { May cause drowsiness }\end{array}$ & $71-36-3$ & 31.09 \\
\hline Cotinine & Warning & $\begin{array}{l}\text { Acute Toxicity } 4 \\
\text { Skin Irritation } 2 \\
\text { Eye Irritation } 2 \mathrm{~A} \\
\text { STOT SE } 3 \\
\text { Harmful if swallowed } \\
\text { May cause respiratory irritation }\end{array}$ & $486-56-6$ & 27.66 \\
\hline 5-Methyl-2-heptanol & Danger & $\begin{array}{l}\text { Acute Toxicity. } 3 \\
\text { Harmful if swallowed }\end{array}$ & $54630-50-1$ & 25.07 \\
\hline 1-Methoxy butane & Danger & Flammable liquid & $628-28-4$ & 24.8 \\
\hline 3-(3,4-dihydro-2H-pyrrol-5-yl) pyridine & Warning & $\begin{array}{l}\text { Acute Toxicity } 4 \\
\text { Skin Irritation } 2 \\
\text { Eye Irritation } 2 \text { A } \\
\text { STOT SE } 3 \\
\text { Harmful if swallowed } \\
\text { May cause respiratory irritation }\end{array}$ & $532-12-7$ & 22.44 \\
\hline $\begin{array}{l}\text { 1-(4-pyridinylmethyl)-1H-pyrazol-5- } \\
\text { amine }\end{array}$ & Danger & $\begin{array}{l}\text { Acute Toxicity. } 3 \\
\text { Harmful if swallowed }\end{array}$ & $3524-31-0$ & 18.12 \\
\hline 2,3-dihydro-1H-inden-1-one & None & None & $83-33-0$ & 17.01 \\
\hline 2-methyl 2-pentanol & Warning & $\begin{array}{l}\text { Flammable liquid } 3 \\
\text { Skin Irritation } 2 \\
\text { Eye Irritation 2A } \\
\text { STOT SE } 3 \\
\text { May cause respiratory irritation }\end{array}$ & $590-36-3$ & 14.99 \\
\hline 3-Ethyl-5-hexen-3-ol & Danger & $\begin{array}{l}\text { Skin Irritation } 2 \\
\text { Eye Damage } 1 \\
\text { STOT SE } 3 \\
\text { Acute Aquatic Toxicity } 3 \\
\text { Chronic Aquatic Toxicity } 3 \\
\text { May cause respiratory irritation }\end{array}$ & $1907-46-6$ & 8.79 \\
\hline 6-Methyl-1,2,3,4-tetrahydroquinoline & Warning & $\begin{array}{l}\text { Acute Toxicity } 4 \\
\text { Skin Irritation. } 2\end{array}$ & $91-61-2$ & 8.79 \\
\hline
\end{tabular}


Table 2 Chemical by-products found in reference aerosol (Continued)

\begin{tabular}{ll}
\hline & $\begin{array}{l}\text { Eye Irritation 2A } \\
\text { STOT SE 3 } \\
\text { Harmful if swallowed } \\
\text { May cause respiratory irritation }\end{array}$ \\
$\begin{array}{l}\text { 1-(p-Toluidio)-1-deoxy-beta-d- } \\
\text { idopyranose }\end{array}$ & NF \\
\hline $\begin{array}{l}\text { S/N ratio }=\text { Signal-to-Noise ratio } \\
\text { TIC }=\text { Total lon Chromatogram } \\
\text { STOT SE }=\text { Specific Target Organ Toxicity, Single Exposure } \\
\text { NF }=\text { Not found }\end{array}$ &
\end{tabular}

e-liquid residue on its heating element (Fig. 3). Although the e-cigarette was operating normally without obvious signs of a mechanical failure (e.g. reduction of aerosol production, e-liquid leaking or flooding, or gurgling noise), we were now able to detect lead (0.097 \pm $0.003 \mathrm{mg} / \mathrm{L})$ and manganese $(0.001 \pm 0.000 \mathrm{mg} / \mathrm{L})$ from $7.5 \mathrm{~L}$ (150 puffs) aerosol.

\section{Discussion}

We developed an e-liquid Reference Material and characterized its physicochemical properties to enhance the reproducibility and promote independent verification in e-cigarette research.

Our results show that a highly pure and accurate eliquid standard can be formulated when a proper Standard Operating Procedure is implemented (Fig. 1). Our homogeneity and stability tests revealed that the e-liquid RM is stable in room temperature up to 1 week, homogeneous up to 2 weeks and stable up to 3 days in our defined operating condition ( $(21$ to 50$) \mathrm{C}^{\circ}, 2.8 \Omega$ heating element and $3.6 \mathrm{~V}$ tank system). However during the e-liquid preparation process, propylene glycol and glycerin are likely to adhere to pipette tips, mixers, and containers due to their moderate and high viscosity (0.042 Pa.s and 1.412 Pa.s, respectively) properties. This viscosity may introduce unintended errors in compositional concentration reports, especially when volumetric measurements are used.

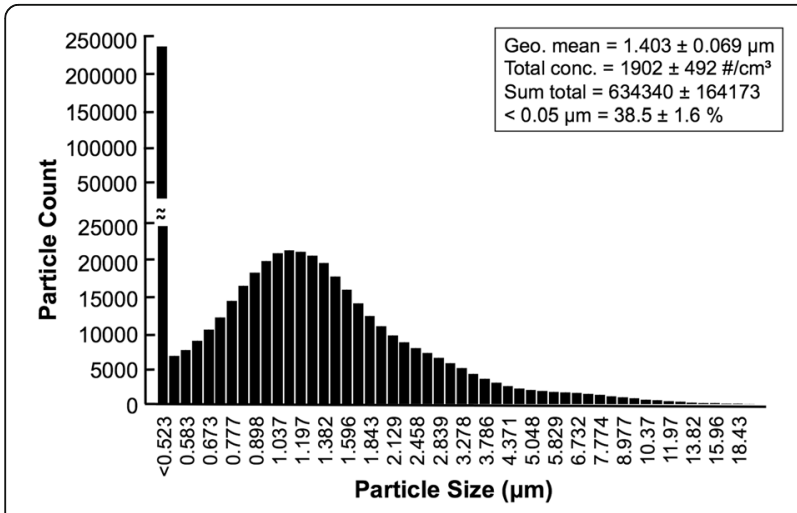

Fig. 2 Particle distribution of reference aerosol (20 s sampling schedule, $1.0 \mathrm{~L} / \mathrm{min}$ sample flow rate)
The gravimetric preparation described here decreases those errors.

The present study has several limitations. We observed a color change in the reference e-liquid at day 7 which led us to presume that oxidation of nicotine was taking place. Although online e-cigarette forums report some ecigarette users "age" their e-liquids by a process called "steeping", nicotine oxidation usually is associated with reduced subjective flavor/taste. However biological effects of oxidized e-liquids, if any, are not known at this point. Future studies should evaluate (i) how the oxidation affects physicochemical properties of e-liquids, (ii) if there are any adverse health effects associated with oxidized eliquids and aerosol generated from them, (iii) how oxidized e-liquids interact with metal components of ecigarettes (e.g. heating element), and (iv) how the perception of flavors changes with the oxidation process. Future studies should also examine if storing the e-liquid RM at cooler temperature (e.g. $-20 \mathrm{C}$ freezer), away from light and moisture will extend its overall service life.

Caution should be taken when interpreting the chemical by-products and metals found in the aerosol as shown in Table 2. Globally Harmonized System (GHS) signal words and hazard classification are based on high concentration and/or repeated exposures of those chemicals. Quantification of the chemical by-products should be considered prior to definitive hazard assessment of e-cigarette aerosol. It is important to point out that metals detected in the aerosol are originating from the e-cigarette device and not from the reference e-liquid. Currently, there is no objective way of determining a lifespan of e-cigarettes. Although online e-cigarette forums recommend replacing the atomizer at the first sign of a mechanical failure or metal taste, the sensitivity of taste varies among individuals, and the level of metal exposure could already be critical if the user can taste metal in the e-cigarette aerosol. Moreover, types of metals used to make heating elements (e.g. nickel, iron-chromium-aluminum, stainless steel, titanium or nickel-chromium), how devices are used (e.g. exposures to rapid heating and cooling cycles), and how heating elements are cleaned (e.g. dry burning or chemical washing) may accelerate the degradation process of e-cigarette devices and heating elements. Studies should further investigate 


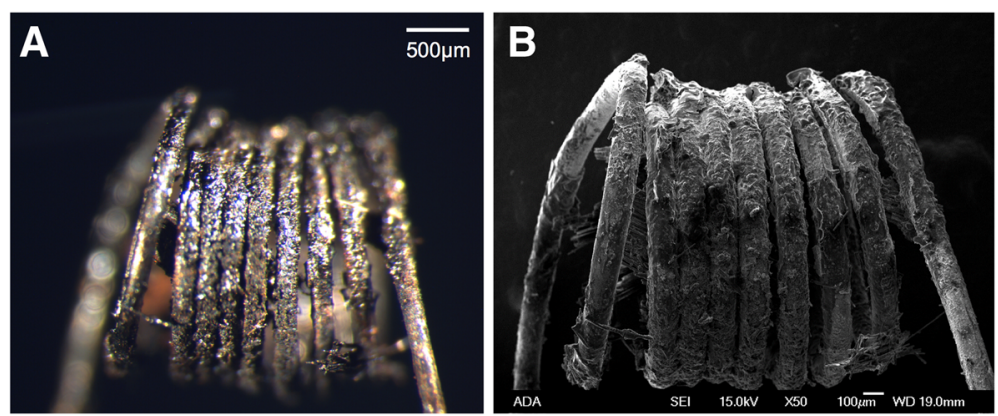

Fig. 3 A heating element after $20 \mathrm{~h}$ of use $\mathbf{a}$ the image was taken by using a stereoptical light microscope (bar = 500 $\mu$ m) and, $\mathbf{b}$ by a scanning electron microscope $($ bar $=100 \mu \mathrm{m})$

the level of metals as a function of time and usage of e-cigarettes.

Furthermore, the authors acknowledge that the quality and quantity of chemical by-products in e-cigarette aerosol will depend on the device and conditions used to generate the aerosol. New devices are constantly being introduced to the general public with updated technologies (e.g. pyrex tubes, bottom coils, adjustable airflow, sub-ohm heating elements, etc). It will be very difficult to test all of them within a short time frame which reinforces the need for development of standard testing devices and reference materials.

Despite limitations, this study provides insight into why inconsistencies in material composition and concentration are observed in many commercially available e-liquids. The current e-cigarette research experimental designs could also benefit from having standards such as the eliquid RM described here. The e-liquid RM can be used to check the quality and metrological traceability of commercial e-liquids during a pre-market testing period, instrument calibration, toxicological risk assessment, and safety and efficacy of e-cigarette devices. Ultimately universal ecigarette testing methods based on a rigorous consensus process from academia, the industry and regulatory government agencies will contribute to understanding and assessing the health risks of e-cigarettes, protection of public safety and promote dissemination of scientifically relevant information in a timely manner.

\section{Conclusions}

A high quality e-liquid RM was formulated by adopting the ISO Guide 35:2006E. Physicochemical properties of the reference e-liquid and its aerosol were determined under conditions closely resembling human modeling of e-cigarette puffing topography. Our results indicate that the reference e-liquid may be used as a part of universal e-cigarette testing methods to enhance chemical, physical, and biological evaluations of e-liquids and e-cigarette devices. Our efforts are consistent with the recent U.S. Food and Drug Administration (FDA) "Deeming
Tobacco Products Amendment" (Docket No. FDA-2014N-0189) and European Union Tobacco Products Directive (2014/40/EU) which in unity calls for better e-cigarette regulations, including transparency in manufacturing and increasing the quality of e-liquids and the safety of e-cigarette devices. The use of universal e-cigarette testing methods and its strategic implementation warrant further study.

\section{Abbreviations}

ABS: Acrylonitrile butadiene styrene; ACS: American chemical society; ADA: American dental association; APS: Aerodynamic particle sizer; BSI: British standards institution; CDC: U.S. Centers for disease control and prevention; EU: European Union; FDA: U.S. Food and Drug Administration; GC: Gas chromatography; GC-MS: Gas chromatography - mass spectrometry; GHS: Globally harmonized system of classification and labeling of chemicals; GUM: ISO Guide to the expression of uncertainty in measurement; HPHCs: Harmful and potentially harmful constituents; HPLC-UV: High performance liquid chromatography - ultraviolet vis absorbance detector; ICP-OES: Inductively coupled plasma - optical emission spectrometry; ISO: International standards organization; MS: Mass spectrometry; MSDS: Material safety data sheet; NIST: U.S. National institute of standards and technology; PPB: Parts per billion; PTFE: Polytetrafluoroethylene; RM: Reference material; RPM: Revolutions per minute; S/N: Signal-to-Noise ratio; SD: Standard deviation; SOP: Standard operating procedure; TIC: Total ion chromatogram

\section{Acknowledgments}

The authors thank Bruce Benner Jr. (NIST) for his insight and helpful discussion regarding Reference Material (RM) development. The authors thank Drago Skrtic, Thomas C. Hart and Gretchen Duppins for critically reviewing the manuscript, Christine Dillon for her administrative support and Magdalena Navarro for translating the AFNOR document. The authors acknowledge National Institute of Standards and Technology (NIST) Department of Commerce for sharing core facilities and high-precision analytical instruments through CRADA. The authors thank the NIST Summer Undergraduate Research Fellowship (SURF) program.

\section{Funding}

This work was supported by the ADA Foundation (Intramural - Clinical Research).

\section{Availability of data and materials}

Authors have provided detailed instructions on how to formulate and appropriately use the e-liquid reference material in the main text. Should readers need further assistance, they can refer to www.adafoundation.org for more information. Certain commercial equipment, instruments, and materials are identified in this paper to describe the experiments performed. Such identification does not imply recommendation or endorsement by the ADA Foundation and/or the National Institute of Standards and Technology, nor does it imply that the materials or equipment identified are necessarily the best available for the purpose. 


\section{Authors' contributions}

JJK took a lead role in the study concept and design, and write-up of the manuscript. NS, WT, DR, and AG prepared the samples and reference materials, and performed homogeneity and stability tests. SF quantified nicotine and metal concentrations. IS and JW characterized chemical properties of the aerosol. JO characterized physical properties of the aerosol. All authors had full access to all data in the study and take responsibility for the integrity of the data and the accuracy of the data analysis. All authors read and approved the final manuscript.

\section{Competing interests}

The authors declare no conflicts of interest.

\section{Consent for publication}

Not applicable.

\section{Ethics approval and consent to participate}

Not applicable.

\section{Author details}

${ }^{1}$ Clinical Research, Volpe Research Center, ADA Foundation, 100 Bureau Drive Stop 8546 NIST, Gaithersburg, MD 20899, USA. ${ }^{2}$ School of Engineering, University of Maryland College Park, College Park, MD, USA. ${ }^{3}$ Molecular Characterization and Analysis Complex, University of Maryland Baltimore County, Baltimore, MD, USA. ${ }^{4}$ Department of Biomedical Engineering, Johns Hopkins University, Baltimore, MD, USA. ${ }^{5}$ Department of Chemistry and Biochemistry, University of Maryland College Park, College Park, MD, USA. ${ }^{6}$ Present address: Food and Drug Administration, Silver Spring, MD, USA.

\section{Received: 3 May 2016 Accepted: 8 February 2017}

\section{Published online: 16 February 2017}

\section{References}

1. Pisinger C, Dossing M. A systematic review of health effects of electronic cigarettes. Prev Med. 2014;69:248-60.

2. Johnston LD, O'Mally PM, Miech RA, et al. Monitoring the future. National survey results on drug use: 1975-2014 Overview key findings on adolescent drug use. 2014. http://www.monitoringthefuture.org/pubs/monographs/ mtf-overview2014.pdf . Accessed 29 Mar 2016.

3. World Health Organization. WHO Framework convention on tobacco control electronic nicotine delivery systems. Moscow: Russian Federation; 2014.

4. King BA, Patel R, Nguyen $\mathrm{KH}$, et al. Trends in awareness and use of electronic cigarettes among US adults, 2010-2013. Nicotine Tob Res. 2015;17(2):219-27.

5. Chatham-Stephens K, Law R, Taylor E, et al. Notes from the field: calls to poison centers for exposures to electronic cigarettes - United States, September 2010-February 2014. MMWR. 2014;63(13):292-3.

6. Corey C, Wang B, Johnson SE, et al. Notes from the field: electronic cigarette use among middle and high school students - United States, 2011-2012. MMWR. 2013;62(35):729-30.

7. Tomar SL, Fox CH, Connolly GN. Electronic cigarettes the tobacco industry's latest threat to oral health? JADA. 2015;146(9):651-3.

8. Harvard T.H. Chan School of Public Health and STAT. Americans' perspectives on e-cigarettes. 2015. https://cdn1.sph.harvard.edu/wpcontent/uploads/sites/94/2015/11/Stat-Harvard-Poll-Oct-2015-AmericansPerspectives-on-E-Cigarettes.pdf. Accessed 29 Mar 2016.

9. American Lung Association. Statement on e-cigarettes. 2015. http://www. lung.org/our-initiatives/tobacco/oversight-and-regulation/statement-on-ecigarettes.html. Accessed 29 Mar 2016.

10. Schoenborn CA, Gindi RM. Electronic cigarette use among adults: United States. 2014. October 2015. http://www.cdc.gov/nchs/products/databriefs/ db217.htm. Accessed 27 Sept 2016.

11. Filippidis FT, Laverty AA, Gerovasili $V$, et al. Two-year trends and predictors of e-cigarette use in 27 European Union member states. Tob Control. 2016;23 Suppl 0:iii1-7.

12. Action on Smoking and Health. Use of electronic cigarettes (vapourisers) among adults in Great Britain. 2016. http://www.ash.org.uk/files/documents/ ASH_891.pdf. Accessed 27 Sept 2016.

13. Lindblom EN. Effectively regulating e-cigarettes and their advertising and the first amendment. Food Drug Law J. 2015;70(1):55-92.
14. Pearson $\mathrm{J}$, Richardson A, Niaura RS, et al. E-cigarette awareness, use, and harm perceptions in US adults. Am J Public Health. 2012;102(9):1758-1766.

15. Zhu SH, Sun JY, Bonnevie E, et al. Four hundred and sixty brands of ecigarettes and counting: implications for product regulation. Tob Control. 2014;23 Suppl 3:iii3-9.

16. Huang, Y. E-Cigarettes: China's next growth industry. May 2014. http://www. forbes.com/sites/yanzhonghuang/2014/05/27/e-cigarettes-chinas-nextgrowth-industry/\#722746db67b4. Accessed 27 Sept 2016.

17. Brown CJ, Cheng JM. Electronic cigarettes: product characterisation and design considerations. Tob Control. 2014;23 Suppl 2:ii4-10.

18. Callahan-Lyon P. Electronic cigarettes: human health effects. Tob Control. 2014;23 Suppl 2:ii36-40.

19. Orr MS. Electronic cigarettes in the USA: a summary of available toxicology data and suggestions for the future. Tob Control. 2014;23 Suppl 2:ii18-22.

20. Goniewicz ML, Kuma T, Gawron M, et al. Nicotine levels in electronic cigarettes. Nicotine Tob Res. 2013;15(1):158-66.

21. Hutzler C, Paschke M, Kruschinski S, et al. Chemical hazards present in liquids and vapors of electronic cigarettes. Arch Toxicol. 2014;88(7):1295-308.

22. Trehy ML, Ye W, Hadwiger ME, et al. Analysis of electronic cigarette cartridges, refill solutions, and smoke for nicotine and nicotine related impurities. J Liq Chromatogr Relat Technol. 2011;34(14):1442-58.

23. Goniewicz ML, Knysak J, Gawron M, et al. Levels of selected carcinogens and toxicants in vapour from electronic cigarettes. Tob Control. 2014;23(2):133-9.

24. Cobb NK, Byron MJ, Abrams DB, et al. Novel nicotine delivery systems and public health: the rise of the "e-cigarette". Am J Public Health. 2010;100(12):2340-2.

25. Cameron JM, Howell DN, White JR, et al. Variable and potentially fatal amounts of nicotine in e-cigarette nicotine solutions. Tob Control. 2014:23(1):77-8.

26. Cheah NP, Chong NW, Tan J, et al. Electronic nicotine delivery systems: regulatory and safety challenges: Singapore perspective. Tob Control. 2014;23(2):119-25.

27. Etter JF, Zather E, Svensson S. Analysis of refill liquids for electronic cigarettes. Addiction. 2013;108(9):1671-9.

28. Hadwiger ME, Trehy ML, Ye W, Moore T, Allgire J, Westenberger B. Identification of amino-tadalafil and rimonabant in electronic cigarette products using high pressure liquid chromatography with diode array and tandem mass spectrometric detection. J Chromatogr A. 2010;1217(48):7547-55.

29. Department of Health. Therapeutic Goods Administration. Australian Government. Liquid nicotine and personal importation for use in electronic cigarettes. 2014. https://www.tga.gov.au/behind-news/liquid-nicotine-andpersonal-importation-use-electronic-cigarettes. Accessed 27 Sept 2016.

30. European Comission. The Tobacco Products Directive (2014/40/EU). 2014 http://ec.europa.eu/health/tobacco/docs/dir_201440_en.pdf. Accessed 27 Sept 2016.

31. U.S. Food and Drug Administration. New regulations for e-cigarettes, cigars, and all other tobacco products. Family smoking prevention and tobacco control act. 2016. http://www.fda.gov/TobaccoProducts/Labeling/ RulesRegulationsGuidance/ucm394909.htm. Accessed 27 Sept 2016.

32. Cheng T. Chemical evaluation of electronic cigarettes. Tob Control. 2014;23 Suppl 2:ii11-17.

33. World Health Organization. Water quality monitoring: a practical guide to the design and implementation of freshwater quality studies and monitoring programmes. 1996. http://www.who.int/water_sanitation_ health/resourcesquality/wqmchap6.pdf?ua=1. Accessed 29 Mar 2016.

34. Jablonski JE, Schlesser JE, Mariappagoudar P. HPLC-UV method for nicotine, strychnine, and aconitine in dairy products. J Agric Food Chem. 2006;54(20):7460-5.

35. Behar RZ, Hua M, Talbot P. Puffing topography and nicotine intake of electronic cigarette users. PLoS One. 2015;10(2):e0117222.

36. Skoog DA, West DM, Holler FJ. Fundamentals of analytical chemistry. 7th ed. Fort Worth: Saunders College Pub.; 1996. 